AperTO - Archivio Istituzionale Open Access dell'Università di Torino

\title{
Performances of the scanning system for the CNAO center of oncological hadron therapy
}

\section{This is the author's manuscript}

Original Citation:

Availability:

This version is available http://hdl.handle.net/2318/68790

since

Published version:

DOI:10.1016/j.nima.2009.11.068

Terms of use:

Open Access

Anyone can freely access the full text of works made available as "Open Access". Works made available under a Creative Commons license can be used according to the terms and conditions of said license. Use of all other works requires consent of the right holder (author or publisher) if not exempted from copyright protection by the applicable law. 


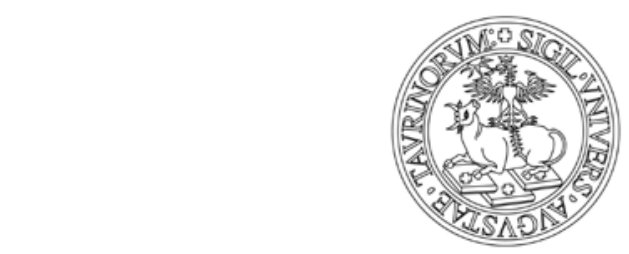

\section{UNIVERSITÀ DEGLI STUDI DI TORINO}

1

2

3

4

5

6
This Accepted Author Manuscript (AAM) is copyrighted and published by Elsevier. It is posted here by agreement between Elsevier and the University of Turin. Changes resulting from the publishing process - such as editing, corrections, structural formatting, and other quality control mechanisms - may not be reflected in this version of the text. The definitive version of the text was subsequently published in Nuclear Instruments and Methods in Physics Research A 613 (2010) 317-322 doi:10.1016/j.nima.2009.11.068

You may download, copy and otherwise use the AAM for non-commercial purposes provided that your license is limited by the following restrictions:

(1) You may use this AAM for non-commercial purposes only under the terms of the CC-BY-NC-ND license.

(2) The integrity of the work and identification of the author, copyright owner, and publisher must be preserved in any copy.

(3) You must attribute this AAM in the following format: Creative Commons BY-NC-ND license (http://creativecommons.org/licenses/by-nc-nd/4.0/deed.en), [+ Digital Object Identifier link to the published journal article on Elsevier's ScienceDirect ${ }^{\circledR}$ platform] 
23 S Giordanengo $0^{1 *}$, M Donetti $^{1,2}, \mathrm{~F} \mathrm{Marchetto}^{1}$, A Ansarinejad $^{3}$, A Attili $^{1}, \mathrm{~F}_{\text {Bourhaleb }}{ }^{1,3}, \mathrm{~F} \mathrm{Burini}^{4}, \mathrm{R} \mathrm{Cirio}^{1,3}, \mathrm{P}$

24 Fabbricatore ${ }^{5}$, F Voelker ${ }^{6}, \mathrm{M} \mathrm{A} \mathrm{Garella}^{1}$, M Incurvati ${ }^{4}$, V Monaco ${ }^{1,3}$, J Pardo ${ }^{1,7}$, C Peroni $^{1,3}$, G Russo $^{1,3}$, R Sacchi $^{1,3}, \mathrm{G}$ 25 Taddia $^{4}$, A Zampieri $^{1}$.

27 (1) INFN (Istituto Nazionale di Fisica Nucleare), Torino, IT

28 (2) CNAO (Centro Nazionale di Adroterapia Oncologica) Foundation, Milano, IT

29 (3) Department of Physics, University of Torino, Torino, IT

$30 \quad$ (4) OCEM SPA, Bologna, IT

31 (5) INFN (Istituto Nazionale di Fisica Nucleare), Genova, IT

32 (6) CERN, Geneve, $\mathrm{CH}$

33 (7) Now at School of CancerStudies, University of Liverpool and Department of Physics, Clatterbridge Centre for 34 Oncology, Merseyside, UK 
37 Abstract. In hadron-therapy one of the most advanced methods for beam delivery is the active scanning technique which uses 38 fast scanning magnets to drive a narrow particle beam across the target. The Centro Nazionale di Adroterapia Oncologica 39 (CNAO) will treat tumours with this technique. The CNAO scanning system includes two identical dipole magnets for horizontal 40 and vertical beam deflection, each one connected to a fast power supply. The dose delivery system exploits a set of monitor 41 chambers to measure the fluence and position of the beam and drives the beam during the treatment by controlling the sequence 42 of currents set by the power supplies. A test of the dynamic performance of the scanning system has been performed using a Hall 43 probe to measure the field inside the magnet and the results are presented in this paper. 


\section{INTRODUCTION}

In contrast to conventional radiotherapy, where electron or photon beams are used, hadron-therapy employs hadrons like protons [1] or carbon ions [2] to irradiate tumour cells. The intrinsic depth-dose deposition curve [3] of charged hadrons has a maximum at a fixed depth in the tissue depending on the particle energy; this property allows for more accurate dose conformity inside the tumour volume as well as lower dose to the surrounding tissues.

The Centro Nazionale di Adroterapia Oncologica (CNAO) [4], presently under construction in Pavia, is a synchrotron based facility to treat deep tumours with protons in the energy range from 60 to $250 \mathrm{MeV}$ and carbon ions from 120 to $400 \mathrm{MeV} / \mathrm{u}$. The CNAO facility includes three treatment rooms, all with horizontal beam lines, and one of them equipped with an additional vertical beam line.

In the active dose delivery technique [5, 2] designed for the CNAO, dose conformation is achieved by changing the beam energy to shift the Bragg peak along the beam direction and, for a fixed energy value, by moving the beam across the tumour.

The matching of the dose profile to the tumour volume is obtained with the superposition of spots, i.e. individual elementary static dose applications delivered by a narrow beam of a fixed energy aiming to a specific position inside the tumour. A careful treatment planning assigns a beam fluence to each spot in order to optimize the dose uniformity into the tumour volume. During the treatment the sweeping across the transverse plane is obtained by means of two independent scanning dipoles for horizontal and vertical beam deflection. This process is supervised by a delivery control system which uses beam monitors for the on-line measurement of the beam position and fluence; when the prescribed fluence of a spot is achieved this system steers the beam to the next spot. The fluence delivered during the transition from a spot to the next is ascribed to the spot where the beam lands. This effect, not accounted for by the treatment planning computation, influences the distribution of the actual delivered dose and has to be reduced by minimising the transit time between spots [6, 7]. For this reason the CNAO scanning system has been designed to reach sufficiently high field ramp rate.

This paper reports several measurements intended to evaluate the performance of the scanning dipole and of the power supply built for the CNAO. In particular the ramp speed, on which the dose released during the beam movement depends, and the linearity of the magnet, which affects the precision of the beam positioning, are studied.

\section{THE CNAO SCANNING AND DOSE DELIVERY SYSTEMS}

The scanning system consists of two power supplies connected to two identical dipole magnets for horizontal and vertical beam deflections located at the end of the extraction line at distances ranging between $4.7 \mathrm{~m}$ and $6.8 \mathrm{~m}$ upstream of the isocenter, depending on the line. The CNAO beam lines have been designed to cover a maximum field of $20 \times 20 \mathrm{~cm}^{2}$ at the isocenter corresponding to a maximum bending angle of $21 \mathrm{mrad}$.

A simplified sketch of the horizontal beam line of CNAO is shown in figure 1. Downstream the magnets, a set of monitor chambers are used to measure the beam fluence and position. These chambers are part of the Dose Delivery system (DD), developed by the CNAO Foundation, the Istituto Nazionale di Fisica 
Nucleare (INFN) and the University of Torino. This system also includes the chamber read out and the delivery control which interfaces to the scanning system [8].

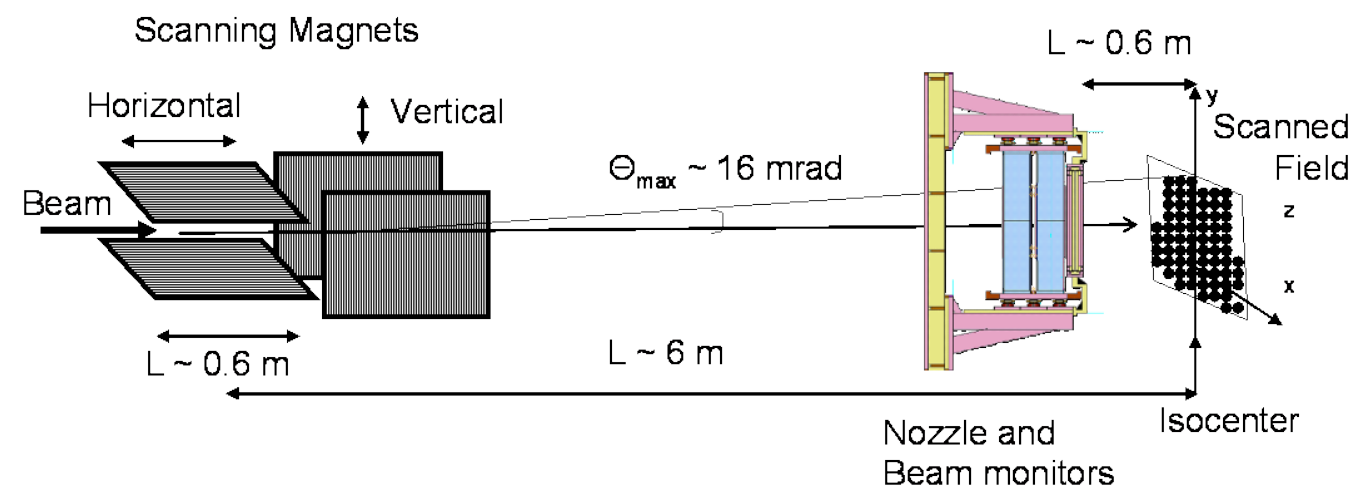

Figure 1: The CNAO horizontal beam line setup.

The sequence of beam positions for each spot, computed by the treatment planning, is translated by the DD into the corresponding magnet currents. When the fluence for a given spot, measured by the monitor chambers, has reached the planned one, the DD transmits the currents corresponding to the next spot position to the power supplies for shifting the beam. During the treatment the DD is designed to provide a feedback correction to the current settings based on the beam position measured by the monitor chambers.

In order to limit the dose inhomogeneity caused by the beam movement between spots within a few percent $(< \pm 2.5 \%$ as clinically required), it has been estimated that the transient time should be less than $200 \mu \mathrm{s}$. This request has to be fulfilled for a large variety of operating conditions. At CNAO the typical step between spots varies from 1 to $3 \mathrm{~mm}$ and the beam rigidity (B $\rho$ ) ranges from $1.14 \mathrm{Tm}$ for $60 \mathrm{MeV}$ protons to $6.38 \mathrm{Tm}$ for $400 \mathrm{MeV} / \mathrm{u}$ carbon ions. The most critical step is $3 \mathrm{~mm}$ with $400 \mathrm{MeV} / \mathrm{u}$ carbon ion beam, which requires a variation of the magnetic field of approximately $80 \mathrm{G}$. The CNAO scanning system has been designed to meet the requirement of a transient time not exceeding $200 \mu$ s for such an extreme condition.

\subsection{Scanning Magnet}

The CNAO scanning magnet has been developed by INFN of Genova in collaboration with the Gesellschaft für Schwerionenforschung (GSI) and 8 units have been built by Danfysik in Denmark. The design of the magnet has been focused to obtain an average field ramp speed of $62 \mathrm{~T} / \mathrm{s}$.

This target has been achieved by using lamination material with high saturation induction, low and uniform coercivity, and steel with minimum amount of impurities and large grain size. Very thin (0.35 $\mathrm{mm}$ ) yoke laminations are glued together to improve the torsion stiffness during the magnet assembly.

The maximum magnetic field of $0.31 \mathrm{~T}$ is achieved with a current of $606 \mathrm{~A}$ circulating in the coils.

The magnet length is $553 \mathrm{~mm}$ and the gap size is $130 \times 140 \mathrm{~mm}^{2}$ with an inner good field region of $120 \times$ $120 \mathrm{~mm}^{2}$. The coil is divided into three sub-coils to optimize the field homogeneity, measured to be better than $0.2 \%$ in the good field region. 
The magnet inductance, calculated from the stored energy, is $4.4 \mathrm{mH}$ and the electrical resistance is 26 $\mathrm{m} \Omega$. The field attenuation $\Delta \mathrm{B}$ decays with a characteristic time constant. Another contribution to the time constant is expected to come from the eddy currents induced in the magnet components (the conductor, the iron lamination and the mechanical structure), causing the field in the gap to be attenuated in ac operations by a quantity $\Delta \mathrm{B}(\mathrm{t})$ depending on the imposed field rate and on the geometrical and electrical characteristics of the magnet components. As an example, in the design phase it was calculated that at a field rate of $62 \mathrm{~T} / \mathrm{s}$ the eddy currents in the iron lamination should cause a maximum field attenuation of $1 \mathrm{G}$ decaying with a time constant of $300 \mu \mathrm{s}$. The eddy currents in the conductor decays with a longer time constant $(3 \mathrm{~ms})$ but with much lower attenuation $(0.3 \mathrm{G})$.

\subsection{Power Supply}

The load of the dipole magnet can be approximated at first order to a R-L circuit with a time constant of 170 ms. This value is large compared to the average time interval required to move the beam between two spots. The time constant can be shortened by three orders of magnitude with the delivering of a large voltage step aborted when the current is close to the required value. The precise adjustment is achieved via smaller voltage steps.

This mechanism has been implemented in the CNAO fast power supplies developed by OCEM S.p.A in collaboration with INFN and CNAO [9].

The power supply is composed of three main modules, sketched in figure 2: a Booster (BO), and two Active Filters (AFs). The BO is a high-voltage-high-current Insulated Gate Bipolar Transistor (IGBT) H-Bridge whereas the AFs are IGBT H-Bridges used in interleaved modulation.

The BO provides a large voltage step $( \pm 660 \mathrm{~V})$ with a ramp speed exceeding $100 \mathrm{kA} / \mathrm{s}$ when a current step larger than $2.5 \mathrm{~A}$ is demanded. The BO is switched-off by a comparator when the current reaches a value close to the set value within \pm 0.6 A. The current is then driven to the final value by the control loops of the AFs. For current steps below 2.5 A only the AFs are used and the BO is short circuited.

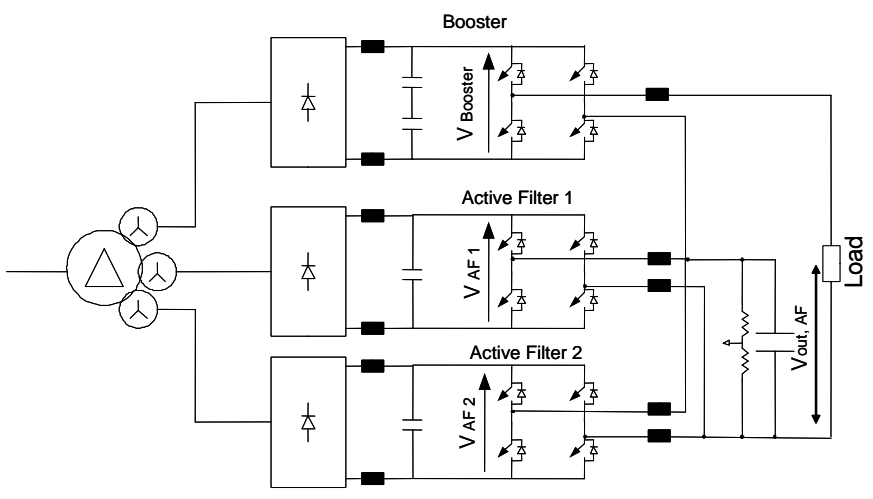

Figure 2: Schematics of the CNAO power supply.

The AFs provide both precise voltage adjustments during the transient and control for steady state current. The precision of the delivered current is $55 \mathrm{~mA}$, corresponding to $100 \mathrm{ppm}$ for $\mathrm{I}_{\max }=550$ A. The AFs control loop sensitivity is $36.4 \mathrm{~mA}$.

The power supply control runs on a National Instruments FPGA mounted on a dedicated PXI crate operating with LabVIEW Real Time operating system.

The connection between the power supply and the magnet is achieved through a $10 \mathrm{~m}$ long shielded cable. 


\section{EXPERIMENTAL SETUP}

\subsection{Hall probe}

A high linearity Hall probe, made by AREPOC s.r.o. [10], has been used to measure the magnetic field in a single point of the scanning dipole. The overall size of the probe is $5 \times 7 \times 1 \mathrm{~mm}^{3}$ with an active area of 0.5 x $1.25 \mathrm{~mm}^{2}$.

The probe output, proportional to the magnetic field in the active area, has been 50 -fold amplified to reach a sensitivity of $22 \mathrm{~V} / \mathrm{T}$. A 16-bit analog-to-digital converter (ADC) has been used to convert the Hall output voltage, the LSB corresponding to $0.7 \mathrm{mV}$ or $0.3 \mathrm{G}$.

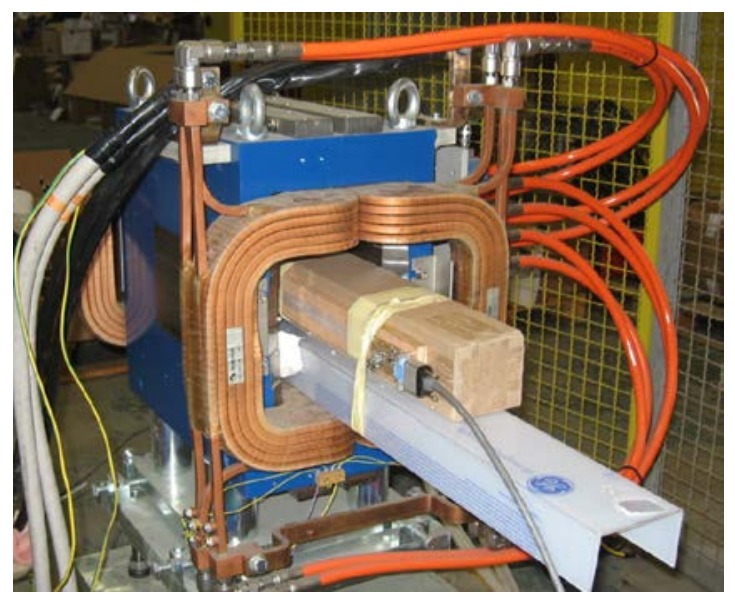

Figure 3: The dipole magnet with the Hall probe in its center.

A picture of the magnet during the tests is shown in figure 3 with the Hall probe mounted on a cardboard box inside the yoke. The probe has been positioned in the center of the magnet with the sensitive area perpendicular to the magnetic field, to maximize the voltage output.

\subsection{On-line control and data acquisition}

A dedicated control and data acquisition system based on the CNAO DD control has been used during this test. This system, hereafter called Dose Delivery DAQ (DDD) is implemented on a National Instrument PXI system featuring a CPU and several PXI modules. The control algorithm runs on a FPGA PXI board NI-7831R equipped with 16-bit ADCs/DACs and several digital inputs/outputs. The FPGA is also connected to a NI I/O DAQ module (DAQ 6534) used as a storage memory.

The control and the data flow between the DDD and the main components of the experimental setup are sketched in figure 4. 


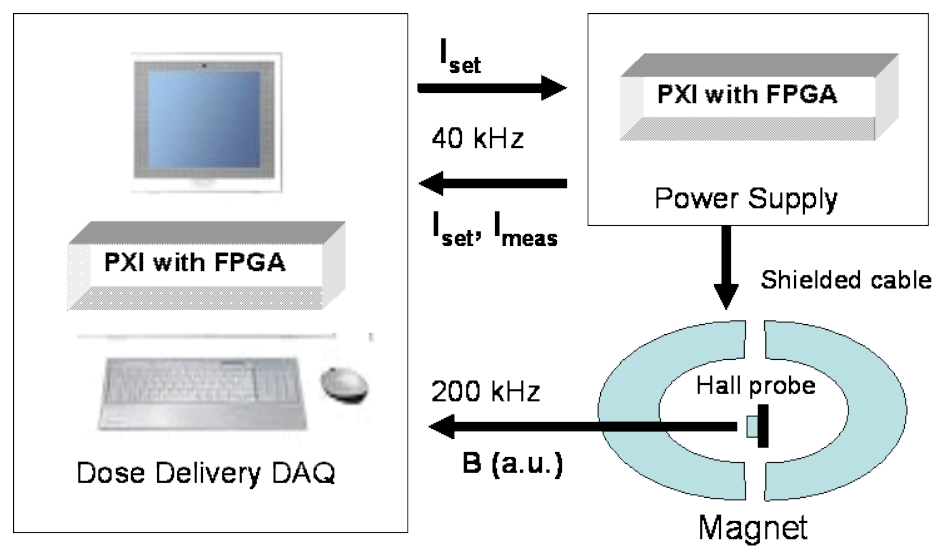

Figure 4: Layout of the experimental setup: components and data flow.

A table with the sequence of currents to be set and the delays between consecutive values is loaded on the DDD. The delays simulates the spot irradiation time assuming a fixed constant beam intensity.

The communication links and protocols implemented are those developed for the CNAO operations. Two 4 Mbaud, 10 meter long, optical links connect the DDD with the power supply by means of custom made optical interfaces. A master-slave communication loop has been implemented, with the DDD as master. The DDD transmits the required current value $\left(I_{\text {set }}\right)$ to the power supply; the latter returns both $I_{\text {set }}$ and the actual measured current $\left(I_{\text {meas }}\right)$, complemented by few control bits. The current values are digitized with a LSB corresponding to $18.3 \mathrm{~mA}$ and the frequency of the loop is $40 \mathrm{kHz}$. The time interval of $25 \mu \mathrm{s}$ is anyway an order of magnitude shorter than the minimum time interval between consecutive spots at CNAO.

The output of the Hall probe is read by an ADC with a frequency of $200 \mathrm{ksample} / \mathrm{s}$, corresponding to a measurement of the magnetic field every $5 \mu \mathrm{s}$. All the measured values ( $\mathrm{I}_{\text {set }}, \mathrm{I}_{\text {meas }}$, ADC counts of the Hall probe) are stored in the memory board and then saved on a hard-disk.

\section{TESTS AND RESULTS}

The experimental setup described in the previous section has been used to test some relevant operational characteristics of the scanning system, namely the linearity and hysteresis of the magnetic field, and its behaviour in ac operations. In particular it is crucial to measure the reaction speed of the system following a DDD request.

Several scans between $I_{\min }(-540 \mathrm{~A})$ and $I_{\max }(+540 \mathrm{~A})$, with increasing and decreasing current steps in the range 1 to $20 \mathrm{~A}$, have been performed. The current limits have been chosen in order to match the maximum field size foreseen at CNAO for carbon ions at maximum energy.

4.1 B measurements: calibration and noise.

Most of the analyses performed to determine the response of the system required only relative $B$ measurements, therefore the absolute calibration of the Hall probe was not strictly necessary. However 
to show the effects of the results on the beam operations, the correspondence between the ADC counts of the Hall probe and the value of the $B$ field has to be assumed.

Table 1 shows the measured B field expressed in ADC counts (second column) for different values of the current (first column). Results were obtained as an average over $1000 \mathrm{~B}$-measurements, taken in $5 \mathrm{~ms}$, for a constant current flowing in the magnet. A common offset of 47 ADC counts has also been subtracted.

\begin{tabular}{|l|l|l|}
\hline I (A) & B (ADC counts) & St. Dev (ADC counts) \\
\hline-539 & -10054 & 3.1 \\
\hline 0 & 0 & 2.5 \\
\hline 540 & +10065 & 3.2 \\
\hline
\end{tabular}

Table 1: Measurements of the Hall probe (mean values and standard deviations) for different currents.

By taking the values measured at the extreme currents, and considering the design value of $0.31 \mathrm{~T}$ maximum field for a current of $606 \mathrm{~A}$, it is derived that one ADC count corresponds approximately to 0.3 $\mathrm{G}$ or $60 \mathrm{~mA}$.

The rms of the measurements, reported in the third column of table 1 , is of about 3 counts, corresponding to approximately $1 \mathrm{G}$. The rms does not depend on the magnetic field, as expected assuming that the noise is induced by the read-out electronics and signal transmission; in particular it results that most of the noise comes from the twisted pair cable connecting the probe to the amplifier and from the amplifier itself.

\subsection{B Linearity}

The linearity of the magnet has been evaluated by analyzing the value of the magnetic field as a function of the delivered current $I_{\text {set }}$. A cyclic scan with the current increasing from $I_{\min }(-540 A)$ to $I_{\max }(+540 A)$ and then decreasing to $I_{\min }$ has been performed with $\pm 10 \mathrm{~A}$ steps and a time interval between two steps of 2 ms.

The results are shown in figure 5, where each point is the average of 200 measurements taken during 1 ms. The error on the average value is less than 0.3 counts. 


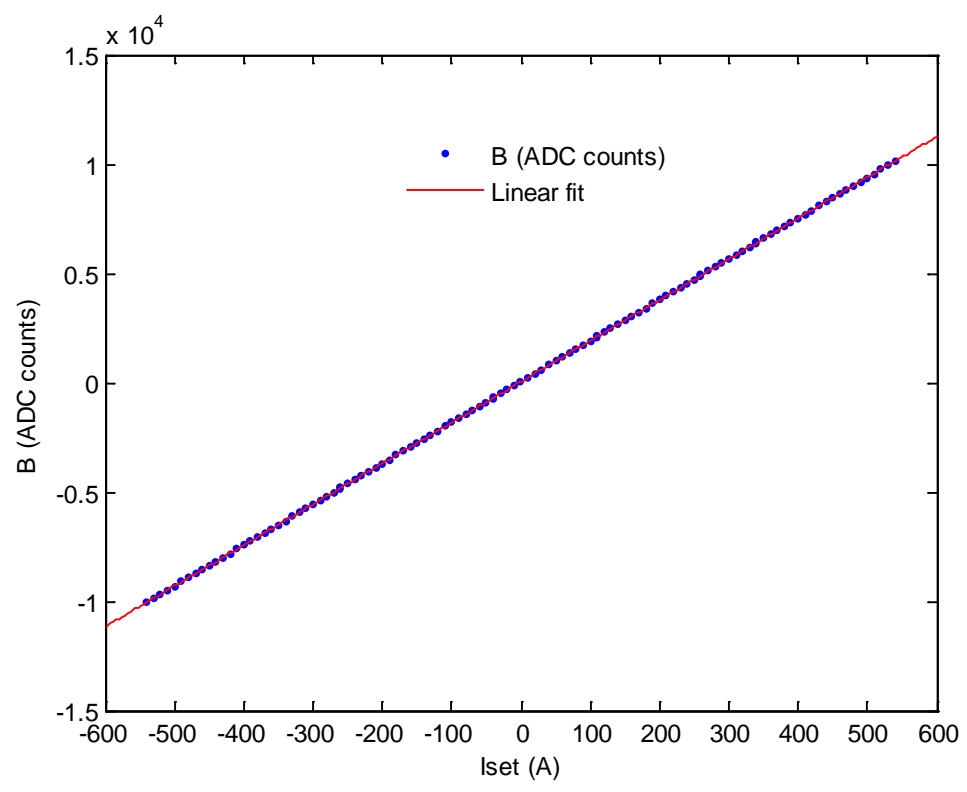

Figure 5: Linearity of the magnet response.

The data points in the range between $-300 \mathrm{~A}$ and $+300 \mathrm{~A}$, where the magnet response is found to be linear, have been fitted with a straight line. The deviations between the measured values and the straight line are shown in figure 6 for the increasing and decreasing scans.

Assuming the correspondence $1 \mathrm{ADC}$ count $=0.3 \mathrm{G}$, the deviation from linearity within the full current range is less than $12 \mathrm{G}$ while the inner linear region is below $2 \mathrm{G}$. This non-linearity effect could be partly related to the Hall probe, which linearity is guaranteed within $0.2 \%$.

Non-linearity effects of the magnet would translate into an error in the beam positioning. A calibration map of the beam position as a function of the current will be used at CNAO to correct for this effect.

The hysteresis, given by the difference between the two set of points in figure 6 , is shown in figure 7 .

The maximum effect is less than $2 \mathrm{G}$. When translated to position, this corresponds to a maximum deviation of approximately $70 \mu \mathrm{m}$ for $\mathrm{C}^{6+}$ and $200 \mu \mathrm{m}$ for protons at the minimum CNAO energies (60 $\mathrm{MeV}$ for protons, $120 \mathrm{MeV} / \mathrm{u}$ for carbon ions). This error could significantly affect the dose distribution; during the clinical operation at CNAO the beam positioning will be controlled and corrected for using the feedback of the beam monitor chambers. The power supply contribution to the hysteresis has been evaluated to be negligible, below 0.2 Gauss. 


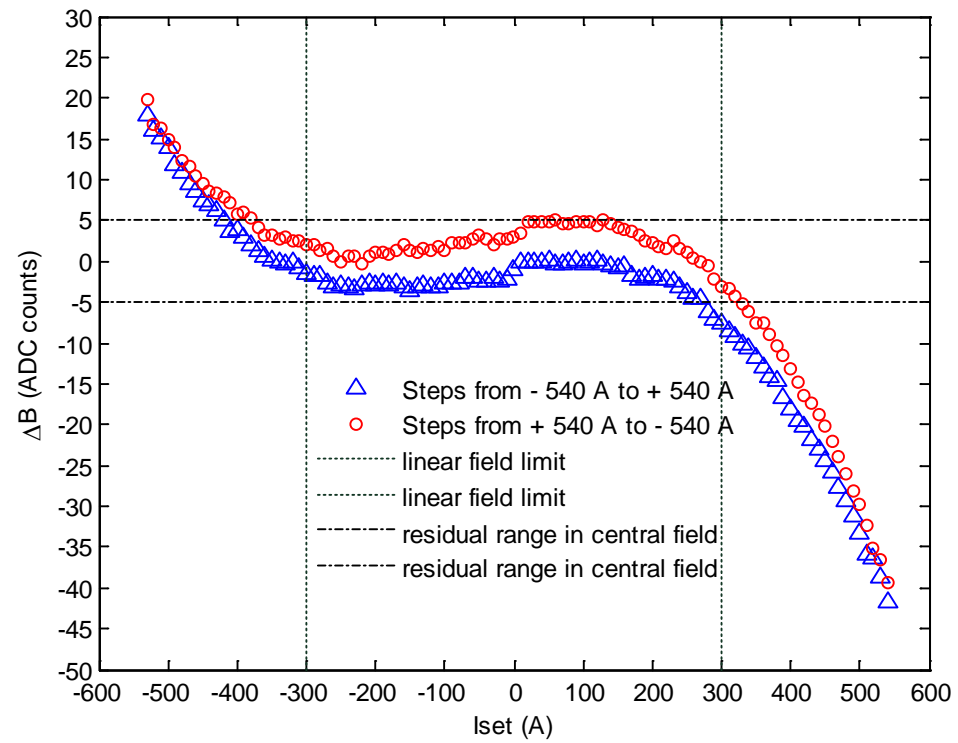

Figure 6: Residuals from linearity for the scans with 10 A steps from -540 A to $540 \mathrm{~A}(\Delta)$ and from $540 \mathrm{~A}$ to $-540 \mathrm{~A}(\mathrm{o})$.

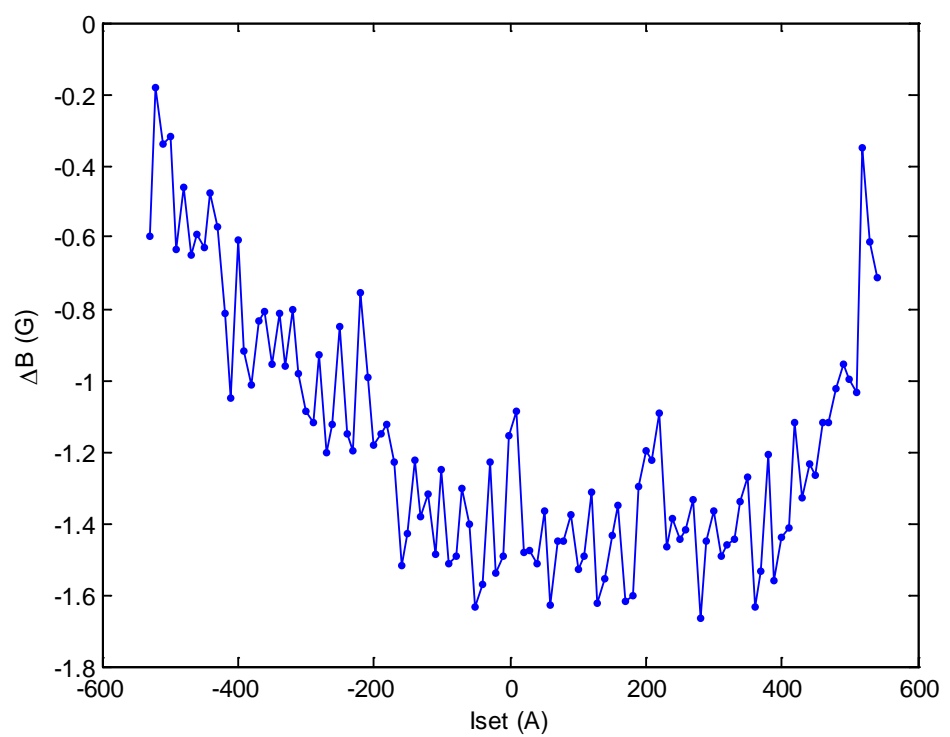

Figure 7: Magnet hysteresis as a function of the current. 


\subsection{Magnet time behavior}

As discussed in the introduction, the performances of the CNAO dose delivery system are strongly influenced by the transient time needed to change the magnetic field of the scanning magnets and perform the beam movement between two spots. Thus several measurements have served to characterize the time performance of the scanning system as a function of the magnet current and the step amplitude.

Two contributions add up to the total time needed to reach the required field: a delay caused by the communication protocol and the transient time of the scanning system. The first contribution is limited by the control loop time interval of $25 \mu$ shile the second contribution can be measured by observing the time behavior of the magnetic field during the ramp. The time delay introduced by the magnetic field measurement system, Hall probe and the amplifier, was estimated to give a completely negligible contribution.

A measurement of the current and magnetic field as a function of time during a $10 \mathrm{~A}$ step from $-480 \mathrm{~A}$ to $-470 \mathrm{~A}$ is shown in figure 8 .

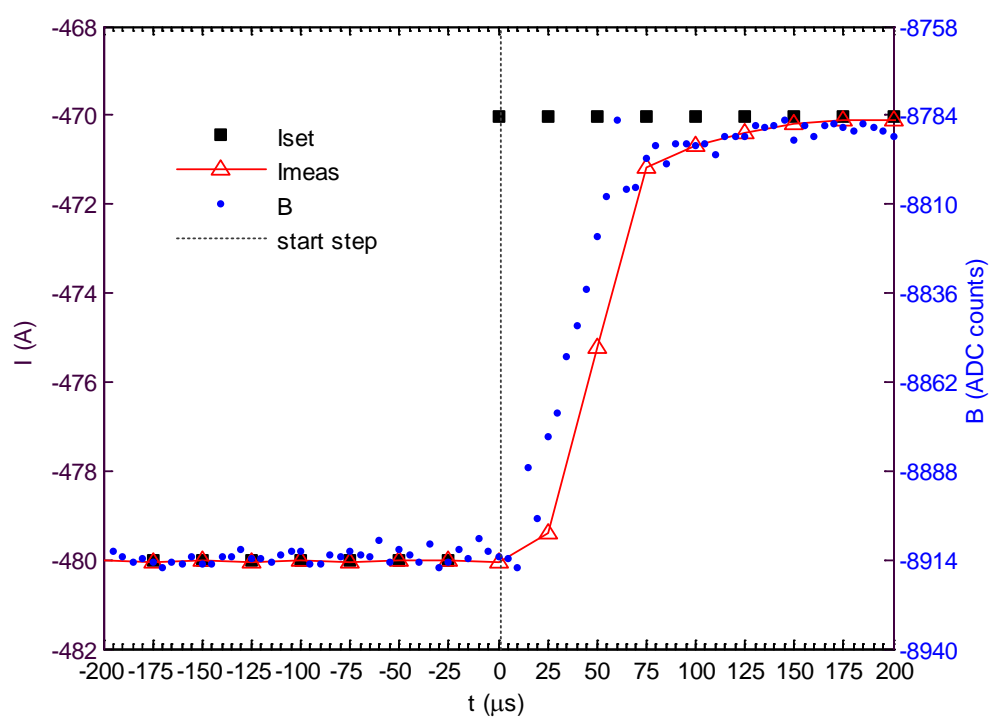

Figure 8: $10 \mathrm{~A}$ step between $-480 \mathrm{~A}$ and $-470 \mathrm{~A}$ (corresponding to a $50 \mathrm{G}$ field variation), for the reference currents $\left(I_{\text {set },},\right)$, the actual currents supplied by the PS $\left(I_{\text {meas }}, \Delta\right)$ and the Hall probe data $(B ;)$.

The reference currents $\left(I_{\text {set }}\right)$ requested by the DDD to the power supply are represented as square dots, the actual currents supplied by the PS ( $\left.I_{\text {meas }}\right)$ are represented by triangles, with a line connecting the measured values. Both $I_{\text {set }}$ and $I_{\text {meas }}$ values are acquired every $25 \mu \mathrm{s}$. The Hall probe data, represented as 
circles, have been acquired every $5 \mu \mathrm{s}$. The delay between the Hall probe data and the measured currents is mainly ascribed to the time required to complete the control loop.

It results from figure 8 that within three current measurements, corresponding to about $50 \mu \mathrm{s}$, most of the magnetic field increase has been accomplished.

The transient time $\Delta \mathrm{t}$, defined as the time interval to accomplish $60 \%$ of the step, from $20 \%$ to $80 \%$ of the step height, is shown in figure 9 as a function of the current $I_{\text {set }}$ for $10 \mathrm{~A}$ steps, both for increasing and decreasing currents. The average value is $\langle\Delta \mathrm{t}\rangle=38.6 \mu \mathrm{s}$ with a rms of $5.8 \mu \mathrm{s}$. This corresponds to a current ramp speed of about $160 \mathrm{kA} / \mathrm{s}$, and to a B-field ramp speed $(\Delta \mathrm{B} / \Delta \mathrm{t})$ of about $85 \mathrm{~T} / \mathrm{s}$. Comparable results were obtained when steps of 15 and $20 \mathrm{~A}$ were applied, for which ramp speeds greater than 150 $\mathrm{kA} / \mathrm{s}$ and $\Delta \mathrm{B} / \Delta \mathrm{t}>75 \mathrm{~T} / \mathrm{s}$ were found.

These results confirm that for $\Delta \mathrm{I}_{\text {set }}>2.5 \mathrm{~A}$ the speed of the magnetic system is well above the design value of $62 \mathrm{~T} / \mathrm{s}$. Considering $90 \%$ or $95 \%$ of a $10 \mathrm{~A}$ step, the transient time increases to 95 or $120 \mu \mathrm{s}$ respectively. It was also observed that $\Delta t$ scales almost linearly with the step amplitude.

For all the steps considered above, the booster of the power supply operates during the ramp. For steps with $\Delta \mathrm{I}_{\text {set }}<2.5 \mathrm{~A}$, for which the booster is short circuited, the scan speed is determined only by the AFs operation which is not linear with the current and depends on the direction of the current variation, especially for steps approaching $I_{\max }$ and $I_{\min }$. This effect is evident in figure 10 where the transient time $\Delta t$ for performing $60 \%$ of $2 \mathrm{~A}$ step is shown as a function of $\mathrm{I}_{\text {set }}$ for increasing and decreasing currents. Three different ranges are considered: currents around $I_{\min }$ around zero and around $I_{\max }$. It results that the transient time is always below $120 \mu \mathrm{s}$ and much better than the design at low currents, which has important implications because the beam is more often scanned in the central part of the field.
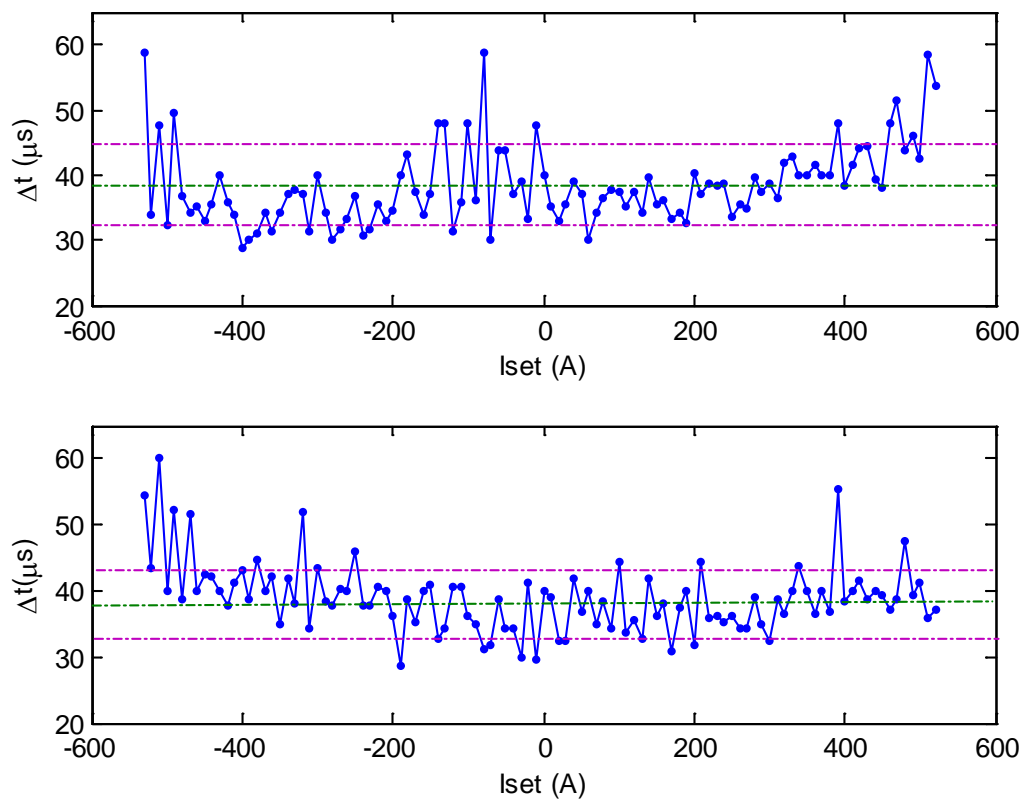
Figure 9. Transient time ( $\Delta \mathrm{t}$ ) between $20 \%-80 \%$ of $10 \mathrm{~A}$ steps versus $\mathrm{I}_{\text {set }}$ for increasing current (upper plot) and decreasing currents (lower plot). The band corresponds to one rms around the average value.

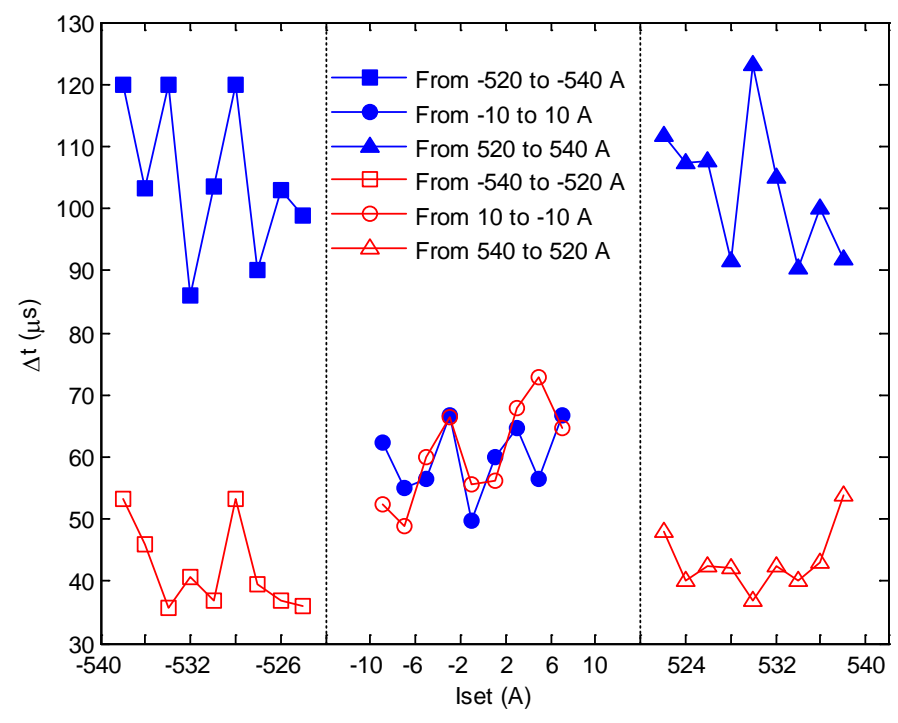

Figure 10: Transient time $(\Delta t)$ between $20 \%-80 \%$ of 2 A steps as a function of $\mathrm{I}_{\text {set }}$ for increasing and decreasing currents.

The uniformity of the transient time was checked by changing the position of the Hall probe within the magnet gap. No dependence on the position has been observed for transient times measured with $2 \mathrm{~A}$ and $10 \mathrm{~A}$ steps.

\section{CONCLUSIONS}

In hadron-therapy with an active dose delivery method, a crucial parameter is the speed of the beam when moving from one spot to the next one. Many efforts have been invested at CNAO in the design of the beam scanning system, i.e. the magnet and the booster driven power supply, to optimize this parameter.

The purpose of the test reported in this paper is the characterization of the scanning system of CNAO. It has been determined that the magnet hysteresis is limited to less than $2 \mathrm{G}$ and that its deviation from linearity is less than $12 \mathrm{G}$ within the full range of operation. The ramp speed of the scanning system has been found to be around $170 \mathrm{kA} / \mathrm{s}$ corresponding to a B-field speed of $85 \mathrm{~T} / \mathrm{s}$ for current steps larger than $2.5 \mathrm{~A}$. 
Within this conditions a step of $10 \mathrm{~A}$, which corresponds to a $3.3 \mathrm{~mm}$ position step for a $270 \mathrm{MeV} / \mathrm{u}$ carbon ion beam, requires around $38 \mu$ s to accomplish from $20 \%$ to $80 \%$ of the increment, while the full step requires $120 \mu \mathrm{s}$. Steps of $2 \mathrm{~A}$, where the booster does not operate, take anyway less than $200 \mu \mathrm{s}$.

All these results presented here are found to be better than the design specifications of CNAO.

\section{REFERENCES}

[1] A R Smith, Vision 20/20 : Proton therapy, Med. Phys. 36 (2), 556-568, (2009)

[2] O Jakel, C P Karger, J Debus, The future of heavy ion radiotherapy, Med. Phys. 35 (12), 5653-5663, (2008)

[3] American Association of Physicists in Medicine (AAPM), Protocol for heavy charged-particle therapy beam dosimetry, AAPM Rep. No. 16 (1986)

[4] www.cnao.it

[5] T Haberer, W Becker, D Shardt, G Kraft, Magnetic scanning system for heavy ion therapy, Nucl. Instrum. Meth A 330, 296 (1993)

[6] J H Kang, J J Wilkens, U Oelfke, Demonstration of scan path optimization in proton therapy, Med. Phys. 34 (9), 3457-3464, (2007)

[7] J Pardo Montero, M Donetti, F Bourhaleb, A Ansarinejad, A Attili, R Cirio, M A Garella, S Giordanengo, N Givehchi, A La Rosa, F Marchetto, V Monaco, A Pecka, C Peroni, G Russo, R Sacchi, Heuristic optimization of the scanning path of particle therapy beams, Med. Phys. 36 (6), 2043-2051, (2009)

[8] S Giordanengo, A Ansarinejad, A Attili, F Bourhaleb, R Cirio, M Donetti, M A Garella, F Marchetto, G Mazza, V Monaco, J Pardo Montero, A Pecka, C Peroni, G Russo, R Sacchi, The CNAO system to monitor and control hadron beams for therapy, IEEE Dresden, Germany 19-25 October 2008 - Nuclear Science Symposium - Conference Record (2008).

[9] M Incurvati, F Burini, M Farioli, G Taddia, M Donetti, S Toncelli, G Venchi, I De Cesaris, C Sanelli, F Voelker, S Giordanengo, F Marchetto, Fast high-power power supply for scanning magnets of CNAO medical accelerator, EPAC 2008, $11^{\text {th }}$ European Particle Accelerator Conferenc Genova (2008).

[10] P Kovác, V Cambel, P Bukva, Measuring the homogeneity of Bi(2223)/Ag tapes by four-probe method and a Hall probe array, Supercond. Sci. Technol. 12 465-471, (1999). 